\title{
Reflets
}

Revue ontaroise d'intervention sociale et communautaire

\section{Les sans-abri et la maladie mentale : perspectives des fournisseurs de services de Sudbury}

\section{Carol Kauppi, Jean-Marc Bélanger et Jennifer Keck}

Volume 7, numéro 1, printemps 2001

Santé mentale et les défis de l'an 2001

URI : https://id.erudit.org/iderudit/026336ar

DOI : https://doi.org/10.7202/026336ar

Aller au sommaire du numéro

Éditeur(s)

Reflets : Revue ontaroise d'intervention sociale et communautaire

ISSN

1203-4576 (imprimé)

1712-8498 (numérique)

Découvrir la revue

Citer cet article

Kauppi, C., Bélanger, J-M. \& Keck, J. (2001). Les sans-abri et la maladie mentale : perspectives des fournisseurs de services de Sudbury. Reflets, 7(1), 48-69. https://doi.org/10.7202/026336ar

Tous droits réservés (C) Reflets : Revue ontaroise d'intervention sociale et communautaire, 2001
Ce document est protégé par la loi sur le droit d'auteur. L'utilisation des services d'Érudit (y compris la reproduction) est assujettie à sa politique d'utilisation que vous pouvez consulter en ligne.

https://apropos.erudit.org/fr/usagers/politique-dutilisation/ 


\section{Les sans-abri et la maladie mentale : perspectives des fournisseurs de services de Sudbury}

Carol Kauppi, Ph.D., Jean-Marc Bélanger, Ph.D. et Jennifer Keck, Ph.D.

École de service social, Université Laurentienne

\section{Introduction}

Il est difficile de comprendre le rapport entre l'état de sans-abri et la maladie mentale, mais aussi de déterminer le nombre de sans-abri atteints de troubles mentaux car on se heurte à divers obstacles : obtention du consentement éclairé des intéressés pour mener les recherches, multiplicité de la population, repérage et suivi des sans-abri qui, de par la nature de leur situation, changent souvent de place. Cependant, les estimations varient de 20 à 50 pour cent (Ontario Medical Association Committee on Population Health 1996). Selon le rapport du Mayor's Homelessness Action Task Force (1999), environ le tiers des sans-abri du Grand Toronto souffrent de troubles mentaux. De fait, des études ont montré récemment que la prévalence des maladies mentales parmi les clients des centres d'accueil de Toronto est deux ou trois fois supérieure à celle constatée dans la population en général, les deux tiers de ces personnes ayant une maladie mentale chronique diagnostiquée (Mental Health Policy Research Group 1997). 
"...beaucoup de

jeunes sans-abri ont une mauvaise santé mentale, en partie parce que la dislocation sociale précoce et l'exposition à la vie dans la rue sont des facteurs propices à la détérioration de la santé mentale..."
Il est essentiel de mener des études longitudinales pour comprendre le lien entre la maladie mentale et l'état de sans-abri, et de pousser les recherches pour expliquer les facteurs déterminants et le cheminement complexe qui mènent à la rue. La collecte d'informations sur les malades mentaux sans-abri est difficile car ces personnes ne s'identifient pas nécessairement par crainte d'être hospitalisées de force, d'être victimes de mauvais traitements et de paraitre suspectes (Mowbray et Bybee 1998). Néanmoins, il est clair, par exemple, que beaucoup de jeunes sans-abri ont une mauvaise santé mentale, en partie parce que la dislocation sociale précoce et l'exposition à la vie dans la rue sont des facteurs propices à la détérioration de la santé mentale (Adlaf et Zdanowicz 1999). En outre, les malades mentaux sont plus susceptibles que les autres sans-abri de vivre dans la rue pendant une période prolongée (Mayor's Homelessness Action Task Force 1999). À son tour, la vie de sans-abri exacerbe généralement la durée et la gravité de la maladie mentale. Les dures conditions de vie des sans-abri les obligent à développer une série de compétences qui les amènent à être hostiles, à ne pas faire confiance aux étrangers, et à veiller surtout à satisfaire leur besoins fondamentaux pour assurer leur survie quotidienne. Dans ces conditions, ils ont peu de chances d'accéder aux services de traitement et de réadaptation qui les aideraient à se soigner. Les malades mentaux sans-abri souffrent plus que la population générale des sans-abri parce qu'ils subissent plus de mauvais traitements, reçoivent moins d'aide et ont plus de problèmes de santé (Ontario Medical Association Committee on Population Health 1996).

Fisk et al. (2000) avancent que les jeunes malades mentaux sans-abri sont victimes de l'interaction de facteurs structuraux et personnels. Le chômage, la pauvreté, la pénurie de logements à prix abordable, la désinstitutionalisation et l'insuffisance des services communautaires de santé mentale sont des facteurs structuraux clés qui se greffent à des facteurs personnels comme les problèmes familiaux, la toxicomanie et les capacités physiques et mentales de la personne. Ces chercheurs ont montré que l'interaction complexe de ces facteurs requiert des services complets d'intervention 
"...la gamme des services offerts aux personnes atteintes d'une grave maladie mentale dans les communautés du Nord de l'Ontario est plus restreinte que dans les régions du sud de la province, malgré des études scientifiques qui montrent que les cas de maladie mentale $y$ sont plus nombreux qu'ailleurs en

Ontario. ") communautaire pour les malades mentaux. La transition de la vie de sans-abri à la vie autonome peut réveiller des souvenirs pénibles du passé car la vie dans la rue les oblige à se préoccuper de leur survie. Le personnel clinique peut offrir un soutien vital aux clients pendant et après la période de transition afin de faciliter l'adaptation aux nouvelles conditions de vie. Cependant, il est aussi vital que le système des services dépasse les modes traditionnels de prestation de services à cette population et réponde à ses besoins en établissant de meilleurs programmes communautaires de soutien et de préparation au logement (Levy 2000). Rapp (1998) a montré que la gestion intensive des cas employant un modèle fondé sur les forces apporte effectivement du soutien aux personnes atteintes de troubles mentaux graves et persistants et les aide à vivre indépendamment dans la collectivité.

De nombreuses études renseignent sur les meilleures pratiques dans le domaine de la santé mentale et sur l'efficacité de services dictés par les consommateurs qui concordent avec les cadres matériels et perceptifs des sans-abri (Clarke Institute of Psychiatry 1998; Culhans 1992). Il est prouvé que les sans-abri ayant de graves troubles mentaux peuvent bénéficier de programmes englobant une vaste intervention communautaire, de services de santé physique et mentale, du soutien au revenu, de la formation professionnelle, de l'hébergement subventionné, de la souplesse dans l'exécution des programmes et de l'intervention permanente des consommateurs.

Malheureusement, la gamme des services offerts aux personnes atteintes d'une grave maladie mentale dans les communautés du Nord de l'Ontario est plus restreinte que dans les régions du sud de la province, malgré des études scientifiques qui montrent que les cas de maladie mentale y sont plus nombreux qu'ailleurs en Ontario. Par exemple, le Nord affiche les taux les plus élevés de suicides (60 pour cent plus élevé que le taux général ontarien) et de tentatives de suicide en Ontario (Public Health Research, Education \& Development Program 2000). C'est aussi dans le Nord de l'Ontario qu'on trouve la plus forte incidence de facteurs liés à la santé mentale, comme la consommation abusive et les excès occasionnels d'alcool. Le taux élevé de pauvreté dans le 
Nord de l'Ontario entrave encore plus la capacité des malades mentaux de vivre indépendamment (van de Sande et al. 1999).

Cet article fait état des résultats d'une étude sur les sans-abri de Sudbury (Ontario) et se concentre sur le lien entre la maladie mentale et la vie de sans-abri au palier local perçu par les fournisseurs de services. Il rapporte aussi les points de vue de ces fournisseurs sur les stratégies visant les sans-abri. En outre, les perceptions des fournisseurs de services et des citoyens sur ce même lien sont comparées aux raisons que les sans-abri euxmêmes ont données pour expliquer leur situation.

\section{Méthodologie}

En raison de la difficulté inhérente à l'étude de cette population, diverses méthodes ont été utilisées pour recueillir des données quantitatives et qualitatives. L'étude s'est déroulée en quatre phases menées simultanément pendant la semaine du 17 au 23 juillet 2000. La phase I a consisté à dénombrer les sans-abri utilisant les refuges, les organismes de services sociaux et d'autres services de soutien de la région de Sudbury, ainsi qu'à recenser les caractéristiques des sans-abri et les raisons de leur situation. La définition du sans-abri employée dans cette étude est celle établie par le Groupe de travail sur les sans-abri dans la région du GrandToronto; elle se fonde sur le travail de Daly (1988) et sur le point de vue que les sans-abri sont "des personnes qui n'ont aucun abri, périodiquement ou temporairement, ainsi que des personnes pour lesquelles le risque de se trouver à la rue dans un avenir immédiat est très grand» (246). L'instrument de collecte des données a été conçu pour obtenir un dénombrement valide et sans double compte de la population sudburoise des sans-abri tout en respectant la vie privée des personnes utilisant les services. Il s'inspire du système national automatisé d'enregistrement des services aux sans-abri (Automated National Client-specific Homeless Services Recording System) (ANCHoR), un système d'information 
(Peressini et al.1996) visant à appuyer la coordination des services aux sans-abri. Le questionnaire a été adapté pour cette étude en vue de recueillir des renseignements sociodémographiques de base sur les personnes utilisant les centres d'accueil et les services connexes, notamment le prénom, l'initiale du deuxième prénom, le nom de famille, la date de naissance, le numéro d'assurance sociale, le sexe, l'ethnicité ou la race, la situation de famille, l'orientation linguistique, les dates d'entrée, de sortie, d'utilisation ou d'abandon des services. Nous avons aussi établi si les personnes étaient assistées sociales et demandé les raisons pour lesquelles elles étaient à la rue. Au cours de réunions en face à face, nous avons donné aux fournisseurs de services des instructions pour recueillir les données auprès de leurs clients. Tous les fournisseurs sollicités, sauf un, ont recueilli les renseignements qui ont servi au dénombrement.

La phase II a consisté à mener une enquête auprès des fournisseurs de services de la région. Le questionnaire visait à rassembler des informations sur les services fournis par les organismes, les dossiers, l'utilisation des lits, les liens entre les organismes, les besoins et les caractéristiques des sans-abri de Sudbury, les facteurs liés à la vie de sans-abri et les stratégies utilisées pour aider les sans-abri de la ville. Les 19 organismes qui ont rempli le questionnaire ont donné un taux de réponse de 79 pour cent. Il s'agissait de refuges, de services alimentaires de secours, d'organismes s'occupant des toxicomanes, et d'autres organismes fournissant des services à des groupes à risque élevé et à des personnes à faible revenu (p. ex., registre du logement, Ontario au travail, Société Elizabeth Fry, Native Friendship Centre, services d'emploi, services policiers, etc.).

Au cours de la phase III, nous avons mené une enquête en personne dans des maisons de quartiers de Sudbury choisis au hasard. Elle avait pour but de sonder l'opinion publique sur les sans-abri et de repérer les «sans-abri occultés» (les "sans domicile fixe») qui trouvent temporairement refuge chez des particuliers. Dans ce volet de l'enquête, les répondants ont été informés que la définition du sans-abri est adaptée de celle utilisée dans l'enquête de DC^MADS (Dennis 1993) : 
Une personne sans-abri ne peut pas se réfugier dans un endroit qu'elle considère chez elle et où elle dort régulièrement. Une personne est sans-abri si :

- elle n'a pas d'endroit qu'elle peut considérer comme son domicile, $\mathrm{OU}$

- son logis n'est ni une chambre, ni un appartement, ni une maison, $O U$

- sa chambre, son appartement ou sa maison ne lui appartient pas, $\mathrm{OU}$

- elle réside à cet endroit quatre jours par semaine ou moins, $O U$

- elle n'est pas organisée pour y dormir régulièrement.

À partir de cartes de Sudbury, nous avons sélectionné plus de la moitié des quartiers de la ville (18 sur 35$)$ afin d'avoir un échantillon représentatif. Le choix s'est fait à l'aide d'un échantillonnage en grappes qui a consisté à choisir un échantillon aléatoire de quartiers puis, à l'intérieur de ceux-ci, un échantillon systématique de résidences personnelles. Les équipes de recherche ont frappé à toutes les cinq portes, toutes les troisièmes rues. De plus, les quartiers pauvres ont été plus échantillonnés à cause du risque élevé d'incidence accrue de sans-abri. Le même questionnaire a été utilisé aux phases I et II afin de recueillir les mêmes renseignements sur les sans-abri occultés que ceux recueillis par les fournisseurs de services pour le dénombrement. Au total, 236 personnes ont répondu au questionnaire. Le taux de réponse à l'enquête menée dans les quartiers a été de 62 pour cent.Avec un taux de 64 pour cent, les femmes ont constitué presque les deux tiers des répondants car ce sont elles en général qui ouvraient la porte aux enquêteurs.

Finalement, la phase IV a consisté en une étude qualitative sur le terrain menée dans les endroits occupés par les sans-abri du centre-ville. Des chercheurs ont accompagné des travailleurs communautaires qui servent les sans-abri, ainsi que les policiers de Sudbury qui patrouillent les endroits occupés par les sans-abri de la ville. Les chercheurs ont observé ces endroits et ont pris des 
notes sur les personnes, les événements, les activités et les environnements qu'ils ont rencontrés. Des notes brèves ont été prises sur place et des notes plus détaillées ont été rédigées immédiatement après chaque observation.

\section{Résultats}

"...les résultats $d u$ dénombrement indiquent que la population totale de sans-abri de Sudbury dépasse de beaucoup les 600 personnes.»

\section{a) Dénombrement des sans-abri}

Le dénombrement des sans-abri a produit le chiffre de 407 utilisateurs différents qui ont utilisé des refuges et des services connexes pendant une semaine en juillet 2000. De plus, 4,2 pour cent des ménages de quartiers à faible revenu ont hébergé temporairement un sans-abri, alors que les répondants des quartiers à revenu moyen ou élevé n'en ont reçu aucun. Une extrapolation de ces résultats à tous les ménages à faible revenu de la région de Sudbury $(\mathrm{n}=6500)$ suggère que la population des sans-abri occultés dépasse les 270 personnes. Par conséquent, les résultats du dénombrement indiquent que la population totale de sansabri de Sudbury dépasse de beaucoup les 600 personnes.

\section{b) Maladie mentale et vie de sans-abri à Sudbury}

En utilisant des estimations prudentes de la prévalence de maladies mentales dans la population des sans-abri ( 25 pour cent), on a établi qu'il y avait environ 167 sans-abri de Sudbury souffrant de troubles mentaux ${ }^{1}$. Cependant, interrogées sur la raison principale pour laquelle elles vivent dans la rue, peu de personnes ( 3 pour cent) ont indiqué la maladie mentale. Elles ont surtout attribué leur sort au chômage, aux salaires peu élevés, aux problèmes d'aide sociale, de logement et de violence conjugale.

Les fournisseurs de services ont orienté environ 5 pour cent des sans-abri vers l'Association canadienne pour la santé mentale.

Les recherches sur le terrain ont montré que des sans-abri doublés de malades mentaux vivent dans les rues de Sudbury, et que les travailleurs communautaires et la police de Sudbury 
connaissent ces hommes, ces femmes et ces jeunes. Les chercheurs les ont observés au centre-ville où ils dorment sur des bancs et le long d'édifices et prennent leur repas du soir à la soupe populaire. Selon la police et les travailleurs communautaires, les endroits populaires pour dormir et se rassembler sont un parc du centreville, les bouches d'air chaud dans une ruelle en arrière d'un édifice public, un endroit sous un pont et des organismes d'aide communautaire (Armée du salut).

\section{b) Avis des fournisseurs de services sur les facteurs ayant amené des malades mentaux de Sudbury à vivre dans la rue}

Nous avons demandé aux fournisseurs de services d'indiquer s'ils étaient d'accord pour dire que diverses caractéristiques et divers facteurs étaient liés à la vie dans la rue. Comme le tableau 1 le montre, presque tout le personnel des organismes pense que la maladie mentale est un facteur clé et même le principal. La comparaison des perceptions des fournisseurs de services et du grand public (recueillies lors de l'enquête dans les quartiers) montre que ce dernier est bien moins enclin à attribuer la faute à la maladie mentale : seulement 3,4 pour cent des répondants à l'enquête menée dans les quartiers ont convenu que la maladie mentale contribue à envoyer les gens à la rue.

Tableau 1 - Avis des fournisseurs de services sur les caractéristiques et les facteurs de risque liés à la vie dans la rue à Sudbury

\begin{tabular}{lc}
\hline Caractéristiques et facteurs & Oui (\%) \\
\hline Personnes atteintes de troubles mentaux & 94,7 \\
Alcooliques ou toxicomanes & 89,5 \\
Adultes & 78,9 \\
Hommes & 78,9 \\
Chômeurs & 78,9 \\
Victimes de violence conjugale ou d'abus sexuels & 73,7 \\
Assistés sociaux & 68,4
\end{tabular}


Tableau 1 - Avis des fournisseurs de services sur les caractéristiques et les facteurs de risque liés à la vie dans la rue à Sudbury (suite...)

\begin{tabular}{lc}
\hline Caractéristiques et facteurs & Oui (\%) \\
\hline Jeunes & 68,4 \\
Membres de minorités visibles ou ethniques & 57,9 \\
Femmes & 52,6 \\
Personnes vivant un divorce ou une séparation & 42,1 \\
Personnes âgées & 42,1 \\
\hline Personnes d'origine européenne & $\mathbf{3 1 , 6}$ \\
\hline
\end{tabular}

De l'avis de la majorité du personnel des organismes, l'alcoolisme et la toxicomanie, le chômage ou l'aide sociale, la violence conjugale ou les mauvais traitements sexuels sont aussi des facteurs qui mènent à la rue. Par surcroît, une grande proportion des fournisseurs de services trouvent qu'un homme adulte court plus de risque qu'une femme ou qu'un jeune; il en va de même des membres d'une minorité visible. Les résultats du dénombrement montrent que seulement 1,7 pour cent des sans-abri de Sudbury appartiennent à des minorités visibles, mais au sein de celles-ci, les Autochtones sont largement représentés ( 25,8 pour cent).

Les répondants ont aussi évalué les facteurs locaux contribuant à jeter des personnes à la rue, surtout celles souffrant de troubles mentaux. Ces facteurs, énoncés dans l'encadré 1, sont d'ordre personnels, environnementaux (en ce qui concerne le système de services de santé mentale) et structuraux. La majorité des fournisseurs de services (plus des deux tiers) ont indiqué neuf facteurs locaux qui contribuent à amener des malades mentaux à la rue. Il s'agit d'une combinaison de facteurs personnels, environnementaux et structuraux (tableau 2). 


\section{Encadré 1 : Facteurs locaux contribuant à amener les malades mentaux à être sans-abri}

\section{Facteurs personnels}

Co-occurence de la maladie mentale et de la toxicomanie

Exposition à des mauvais traitements (physiques ou sexuels)

Mauvaises relations familiales

\section{Facteurs environnementaux (système de santé mentale)}

Planification inadéquate du congé

Limitations des ressources

Pénurie de services communautaires de traitement et de soutien

Pénurie de mécanismes communautaires d'intervention d'urgence

Manque d'attention aux préférences des consommateurs

Manque de coordination entre les systèmes de santé mentale et de traitement de la toxicomanie

\section{Facteurs structuraux}

Pénurie de logements à prix abordable

Insuffisance des prestations d'invalidité

Discrimination

Tableau 2 - Avis des fournisseurs de services sur les facteurs locaux contribuant à amener des malades mentaux à devenir sans-abri ${ }^{1}$

\begin{tabular}{llcc}
\hline Aspect & Facteurs locaux & $\begin{array}{l}\text { D'accord } \\
\mathbf{( \% )}\end{array}$ & $\begin{array}{l}\text { Tout à fait } \\
\text { d'accord } \\
\mathbf{( \% )}\end{array}$ \\
\hline Personnel & $\begin{array}{l}\text { Co-occurrence de la maladie mentale } \\
\text { et de la toxicomanie }\end{array}$ & 33,3 & 61,1 \\
Environnemental & $\begin{array}{l}\text { Pénurie de mécanismes communautaires } \\
\text { d'intervention d'urgence }\end{array}$ & 50 & 43,8
\end{tabular}


Tableau 2 - Avis des fournisseurs de services sur les facteurs locaux contribuant à amener des malades mentaux à devenir sans-abri ${ }^{1}$ (suite...)

\begin{tabular}{|c|c|c|c|}
\hline Aspect & Facteurs locaux & $\begin{array}{l}\text { D'accord } \\
(\%)\end{array}$ & $\begin{array}{l}\text { Tout à fait } \\
\text { d'accord } \\
(\%)\end{array}$ \\
\hline Personnel & Mauvaises relations familiales & 41,2 & 47,1 \\
\hline Environnemental & Limitations des ressources & 61,1 & 22,2 \\
\hline Environnemental & $\begin{array}{l}\text { Pénurie de services communautaires } \\
\text { de traitement et de soutien }\end{array}$ & 52,9 & 29,4 \\
\hline Structural & Pénurie de logements à prix abordable & 43,8 & 37,5 \\
\hline Personnel & $\begin{array}{l}\text { Exposition à des mauvais traitements } \\
\text { (physiques ou sexuels) }\end{array}$ & 37,5 & 37,5 \\
\hline Structural & Discrimination & 50 & 25 \\
\hline Environnemental & Planification inadéquate du congé & 47,1 & 23,5 \\
\hline Structural & Insuffisance des prestations d'invalidité & 37,5 & 6,3 \\
\hline Environnemental & $\begin{array}{l}\text { Manque de coordination entre les } \\
\text { systèmes de santé mentale } \\
\text { et de traitement de la toxicomanie }\end{array}$ & 23,5 & 17,6 \\
\hline Environnemental & $\begin{array}{l}\text { Manque d'attention aux préférences } \\
\text { des consommateurs }\end{array}$ & 26,7 & 13,3 \\
\hline
\end{tabular}

${ }^{a}$ Les points sont énumérés par ordre décroissant du degré d'entente des fournisseurs de services. Celui-ci est calculé en faisant la moyenne des pourcentages des colonnes "D'accord» et «Tout à fait d'accord».

Les trois principales causes sont la conjugaison de la maladie mentale et de la toxicomanie, la pénurie de mécanismes communautaires d'intervention d'urgence, et les mauvaises relations familiales. Il est également largement entendu que les ressources limitées du système de santé mentale, la pénurie de services communautaires de traitement et de soutien, et le manque de logements à prix abordable sont responsables, au palier local, de la situation des malades mentaux sans-abri.

Moins de la moitié des fournisseurs de services de Sudbury attribuent la faute à l'insuffisance des prestations d'invalidité, au 
«...certains fournisseurs

de services ont indiqué

qu'étant donné qu'il

n'existe pas de services

communautaires de suivi

pour les malades

mentaux, les

propriétaires craignent de

leur louer un

appartement. » manque de coordination entre les systèmes de santé mentale et de traitement de la toxicomanie, et au manque d'attention aux préférences des consommateurs.

Des données qualitatives provenant de questions ouvertes posées dans les enquêtes ont fourni des renseignements sur le travail auprès des sans-abri atteints de troubles mentaux. Un problème souvent mentionné est le défaut de prendre les médicaments et de suivre un traitement. Il est souvent impossible de servir les sans-abri ayant des troubles mentaux qui refusent de prendre des médicaments et de voir un médecin. En particulier, les personnes ayant deux types de troubles sont souvent celles qui ne vont pas dans les centres d'accueil. De plus, les fournisseurs de services ont dit que les clients agités peuvent menacer leur sécurité et que les travailleurs n'aident pas ces clients s'ils jugent qu'ils courent un danger.

Au plan du logement, certains fournisseurs de services ont indiqué qu'étant donné qu'il n'existe pas de services communautaires de suivi pour les malades mentaux, les propriétaires craignent de leur louer un appartement. En outre, il semble que le système insuffisant de soutien des malades mentaux mène à des méprises car les troubles de santé mentale peuvent réduire les capacités de certaines personnes de se débrouiller et de résoudre leurs problèmes. Ces types de problèmes produisent des «sansabri récidivistes» qui vivent parfois dans la rue et parfois dans un logement.

\section{c) Avis des fournisseurs de services sur les stratégies de réinsertion sociale des sans-abri}

Les quatre stratégies qui, de l'avis des fournisseurs de services, sont les plus prometteuses pour aborder le problème des sans-abri sont : de meilleurs programmes de soutien, faire face à l'alcoolisme et à la toxicomanie, offrir de meilleurs revenus aux sans-abri, et offrir des options de logement à prix abordable (tableau 3). Trois quarts des fournisseurs de services considèrent également comme des stratégies clés celles qui touchent la violence familiale, l'amélioration des programme d'assistance publique et la réduction des loyers. Plus de la moitié sont également en faveur d'offrir des services d'emploi, 
de simplifier les démarches pour obtenir de l'aide sociale, et d'améliorer la situation des assistés sociaux. Seulement une minorité accorde de l'importance au soutien en cas de divorce ou de séparation pour aider les sans-abri.

Tablean 3 - Avis des fournisseurs de services sur les stratégies de réinsertion sociale des sans-abri a

\begin{tabular}{lcc}
\hline Stratégies & $\begin{array}{l}\text { D'accord } \\
(\mathbf{\%})\end{array}$ & $\begin{array}{l}\text { Tout à fait } \\
\text { d'accord } \mathbf{( \% )}\end{array}$ \\
\hline Offrir de meilleurs programmes de soutien & 31 & 63 \\
Se préoccuper de l'alcoolisme et de la toxicomanie & 25 & 63 \\
Offrir de meilleurs revenus & 31 & 56 \\
Offrir des logements à prix plus abordable & 31 & 56 \\
Se préoccuper de la violence familiale & 38 & 38 \\
Offrir une meilleure assistance publique & 38 & 38 \\
Réduire les loyers & 50 & 25 \\
Offrir des services d'emploi & 40 & 33 \\
Améliorer la situation des assistés sociaux & 25 & 38 \\
Se préoccuper des cas de divorce et de séparation & 20 & 13 \\
\hline
\end{tabular}

${ }^{a}$ Les points sont énumérés par ordre décroissant du degré d'entente des fournisseurs de services. Celui-ci est calculé en faisant la moyenne des pourcentages des colonnes "D'accord» et «Tout à fait d'accord».

Les données qualitatives recueillies lors des enquêtes renforcent le point de vue que le système de santé mentale est inadéquat à Sudbury pour traiter les sans-abri ayant des troubles mentaux. En particulier, les grandes lacunes sont la pénurie de services de santé mentale ouverts jour et nuit et de services d'hébergement de transition. D'autres failles existent dans les services destinés à d'autres groupes, notamment, l'hébergement des familles des malades mentaux qui traversent une crise, des unités de relève pour les membres de la famille, des lits pour les personnes difficiles à placer ou qui font souvent des crises, des lits pour celles atteintes de troubles doubles, et des refuges pouvant accueillir pendant 
l'hiver des femmes atteintes de troubles mentaux. De plus, les personnes ayant des troubles mentaux graves ont besoin de soutien communautaire permanent, comme la gestion de leur cas, un service jugé vital.

Beaucoup de fournisseurs de services ont fait état des problèmes posés par Ontario au Travail qui ont amené des malades mentaux à la rue. Certains ont fait remarquer que la charge de travail des travailleurs sociaux de ce programme est trop lourde et qu'ils ne peuvent pas aider les malades mentaux. Par conséquent, il faut mettre sur pied d'autres services de soutien de sorte que les personnes sans domicile fixe puissent accéder à des prestations d'aide sociale.

Les fournisseurs de services estiment aussi que des travailleurs sociaux affectés au logement devraient travailler avec les malades mentaux pour les aider à trouver un logis permanent, à déménager et à réussir la transition vers un mode de vie stable dans la collectivité. Ces travailleurs pourraient défendre les intérêts de leurs clients en éduquant les propriétaires de logement et le public en général, et en s'efforçant de réduire le stigmate de la maladie mentale. L'obstacle qui empêche les sans-abri de trouver un logement est la tendance des propriétaires à demander des loyers que les assistés sociaux n'ont pas les moyens de payer. Les fournisseurs de services pensent qu'il faut créer des postes de travailleurs sociaux affectés au logement de sorte qu'ils puissent travailler avec les propriétaires et épauler ceux qui acceptent des locataires atteints de troubles mentaux.

Il faut aussi veiller à ce que les sans-abri puissent accéder aux programmes et services en allant les leur offrir sur place, dans la rue, au lieu de s'attendre à ce qu'ils se rendent aux centres où ils sont offerts. Cette souplesse dans la programmation devrait s'étendre à la façon dont les clients sont servis. Les malades mentaux sans-abri doivent être traités comme des personnes uniques et servis en fonction de leurs besoins et de leurs circonstances propres. Il est entendu que «beaucoup de nos [clients] malades mentaux refusent de se faire soigner» et que les troubles anxieux ont une part de responsabilité en ce qu'ils les empêchent d'accéder aux services. Des fournisseurs de services pensent qu'il faut réduire 
«...la pénurie de services en français suggère que la structure actuelle ne répond peut-être pas aux besoins culturels et linguistiques des sans-abri, et peut signifier que les sansabri francophones atteints de troubles mentaux graves ont moins accès aux services de soutien.» les formalités d'accès aux services. D'autres conviennent «qu'il y aura toujours un petit pourcentage de clients qui refuseront ou seront dans l'incapacité d'accéder aux ressources.» Certains ont dit que même en réglant les problèmes systémiques, des malades mentaux et des toxicomanes continueront à être sans-abri.

\section{d) Prise en compte de la langue et de la culture dans les services}

Certains fournisseurs ont dit que les programmes et services actuels destinés aux sans-abri ne sont pas offerts en français et qu'il faut établir des services bilingues. Il convient de souligner qu'on a relevé seulement 11 pour cent de francophones lors du dénombrement des sans-abri, ce qui laisse penser qu'ils sont sous-représentés dans cette population. Si ce chiffre est exact, il faudrait effectuer d'autres études afin de déterminer pourquoi les francophones sont moins susceptibles de finir à la rue. Il importe malgré tout d'étudier la question plus à fond car il est possible que le paramètre ethnique de l'enquête n'ait pas été assez précis ou que les francophones aient tendance à éviter les organismes qui ne les servent pas dans leur langue. Quoi qu'il en soit, la pénurie de services en français suggère que la structure actuelle ne répond peut-être pas aux besoins culturels et linguistiques des sans-abri, et peut signifier que les sans-abri francophones atteints de troubles mentaux graves ont moins accès aux services de soutien. De plus, les résultats du dénombrement montrent clairement que les Autochtones dominent largement dans la population des sansabri de Sudbury dont ils représentent le quart (alors qu'ils représentent moins de 2 pour cent de la population totale). Malgré ces chiffres élevés, il n'existe actuellement pas de centre d'accueil approprié pour servir ce groupe.

\section{Discussion et conclusion}

Les fournisseurs de services de Sudbury qui offrent des programmes et des services aux personnes qui vivent dans la rue 
"À l'exception d'un fournisseur de services, tous pensent que le principal facteur qui mène des malades mentaux à la rue est la maladie mentale conjuguée à la toxicomanie. » considèrent la maladie mentale comme un facteur important. Ils pensent aussi qu'une combinaison de facteurs environnementaux, personnels, structuraux et liés aux services exacerbe l'ampleur du problème chez les malades mentaux. À l'exception d'un fournisseur de services, tous pensent que le principal facteur qui mène des malades mentaux à la rue est la maladie mentale conjuguée à la toxicomanie. Cette affirmation concorde avec les études sur le rapport entre les problèmes de santé mentale et l'alcoolisme et la toxicomanie. En effet, des études américaines ont montré que plus de la moitié des jeunes et des adultes sans-abri présentent à la fois des troubles mentaux et des problèmes d'alcoolisme ou de toxicomanie (Levy 2000; Unger et al. 1997).

Il a été prouvé que les personnes ayant une longue histoire d'alcoolisme ou de toxicomanie ont toute une gamme de problèmes personnels, socio-économiques et de santé physique et mentale (Cox et al. 1998). À l'instar des personnes ayant de graves troubles mentaux, les toxicomanes chroniques sont moins susceptibles de bénéficier des méthodes standard de traitement. Il est prouvé que les malades mentaux sans-abri et les toxicomanes ont en grande partie besoin des mêmes traitements (Oakley et Dennis 1996). Par conséquent, des approches qui profitent aux personnes conjuguant la maladie mentale et la toxicomanie seront probablement aussi efficaces chez les malades mentaux qui ne sont pas toxicomanes.

Plus de 90 pour cent des fournisseurs de services ont souligné le besoin de services communautaires d'intervention d'urgence. Il est évident que les formules standard de prestation de services dans des bureaux et de jour ne conviennent pas aux sans-abri atteints de troubles mentaux graves. Le système des services doit s'éloigner des modèles traditionnels afin de répondre à leurs besoins. De fait, de nombreuses études des meilleures pratiques mettent en évidence les «ingrédients efficaces» des services destinés aux personnes ayant des troubles mentaux graves (Rapp 1998). Les systèmes d'intervention d'urgence constituent un élément clé car ils permettent d'intervenir, en dehors des salles d'urgence et des hôpitaux, en cas de crises découlant d'épisodes de psychose, de problèmes socio-économiques (comme la pauvreté ou la perte 
"...le système de santé mentale ne doit plus se concentrer sur le «client 'non conforme' mais sur le système de soins 'non conforme'»..." du logement), ou de perte des réseaux de soutien social (Clarke Institute of Psychiatry 1998). Cependant, il est clair que la mise en oeuvre de systèmes d'intervention d'urgence exige un accroissement substantiel des ressources, comme l'a fait remarquer la vaste majorité des fournisseurs de services de Sudbury.

Les articles sur les meilleures pratiques soulignent l'importance d'une approche coordonnée de la prestation des services. De fait, la notion de système d'intervention d'urgence englobe une gamme de fournisseurs de services travaillant ensemble pour offrir une série complète d'options permettant de répondre aux besoins particuliers des consommateurs. Selon Rapp (1998), un modèle efficace se concentre sur les forces individuelles, met en évidence les services souhaitables, et intègre ou "enveloppe» les services et les ressources disponibles (y compris les soutiens naturels) autour du client. Pour appliquer ce type de processus, les organismes doivent faire preuve de souplesse, coopérer dans la prestation des services et se détacher des approches traditionnelles.

Il convient de souligner qu'une minorité seulement de fournisseurs de services considère que le manque d'attention aux préférences du consommateur constitue un facteur local qui contribue à mener les malades mentaux à la rue. Ils ont indiqué que trop de sans-abri sont intimidés et se sentent jugés quand ils demandent de l'aide. Le système actuel de services aggrave trop souvent les conditions de vie médiocres des sans-abri, surtout des malades mentaux. Rapp (1998) avance que le protocole traditionnel suivi dans le soutien social comporte le "paradigme de la pathologie» qui a des effets négatifs sur la qualité de vie des malades mentaux et conduit au chômage, à la solitude, à la vie dans la rue, à l'hospitalisation ou à l'emprisonnement. Comme le suggère Culhans (1992), le système de santé mentale ne doit plus se concentrer sur le "client 'non conforme' mais sur le système de soins 'non conforme'» (66). Un système plus axé sur les clients et commandé par les consommateurs répondrait plus efficacement aux besoins des malades mentaux sans-abri.

La plupart des fournisseurs de services conviennent que la pénurie de logements à prix abordable à Sudbury est un problème structural qui accroît le nombre de sans-abri parmi la population 
des malades mentaux. De fait, même si Sudbury arrive en deuxième place des 26 villes canadiennes ayant un fort taux de logements vacants, elle fait aussi partie des cinq villes ontariennes où les loyers sont très élevés (Dunphy et al. 1999). Les données qualitatives provenant de l'enquête menée auprès des fournisseurs de services révèlent que l'allocation de logement incluse dans les prestations d'aide sociale est trop basse pour couvrir le loyer d'un appartement ou d'une chambre. Les fournisseurs de services pensent aussi que les propriétaires fixent intentionnellement les loyers au-dessus du seuil que les prestataires d'Ontario au travail peuvent se permettre, ce qui fait que toute majoration des allocations de logement s'accompagnerait de hausses des loyers. Ils estiment également que les propriétaires agissent de manière discriminatoire envers les bénéficiaires de l'aide sociale en général, et hésitent particulièrement à faire affaire avec des malades mentaux.

Il importe de créer de nouvelles unités de logements sociaux (et d'instaurer d'autres stratégies) afin de fournir un logement décent et abordable à tous les sous-groupes de sans-abri. Cependant, Levy (2000) est en faveur d'un «pré-traitement» des personnes souffrant de troubles mentaux graves, qui les prépare au traitement en leur permettant de reconnaitre leurs problèmes, et leur fournit la motivation d'améliorer leur condition. Pour ce faire, il importe de mettre en oeuvre des initiatives d'intervention communautaire de qualité, des stratégies de réduction des effets négatifs, et une intervention thérapeutique axée sur le client combinée à des solutions de placement qui conduisent graduelle-

"Il est vital de prendre le temps d'établir des relations et de fournir un soutien permanent tout au long $d u$ processus pour que les malades mentaux ne se retrouvent plus dans la rue..." ment à un logement sûr. Il est vital de prendre le temps d'établir des relations et de fournir un soutien permanent tout au long du processus pour que les malades mentaux ne se retrouvent plus dans la rue (Culhans 1992; Fisk et al. 2000; Levy 2000).

L'exposition aux sévices est un facteur personnel qui peut elle aussi mener les malades mentaux à la rue. Comme nous l'avons indiqué plus haut, les études longitudinales sur les sans-abri ne sont pas assez nombreuses et il est difficile de démêler les diverses causes de cette situation dans différentes sous-populations. Cependant, de nombreux auteurs ont décrit le taux élevé de mauvais traitements chez les sans-abri. En particulier, de 40 à 70 pour 
"Les gouvernements fédéral et provincial ont bien alloué de nouveaux subsides en 2000 pour s'occuper des sans-abri, mais la part du lion (80 pour cent) est allée aux grandes villes." cent des femmes sans-abri ont été victimes de sévices physiques ou sexuels avant et pendant la ou les périodes où elles étaient à la rue (Grimm et Maldonado 1995), et plus d'un tiers des adolescents sans-abri ont été victimes d'abus sexuels et, dans la rue, ont été exposés à la violence et à d'autres assauts (Unger et al. 1997). Les services pour les sans-abri atteints de troubles mentaux doivent tenir compte de la forte probabilité que leurs clients ont été victimes de sévices, et comporter des mécanismes pour les aider en leur offrant des services de santé mentale et en les encourageant à y recourir.

Les fournisseurs de services de Sudbury s'entendent pour dire que la combinaison des facteurs locaux liés aux problèmes personnels, aux lacunes du système des services, et aux causes structurales contribuent à mettre des malades mentaux à la rue. Ils estiment que la solution à ces problèmes consiste à améliorer les programmes communautaires de soutien et à régler les questions structurales que sont la pauvreté et la pénurie de logements. À cet égard, des collectivités du nord comme Sudbury ne diffèrent pas de celles du sud de l'Ontario et des États-Unis; les mêmes facteurs et solutions ont été largement discutés dans les articles sur les sans-abri. Malheureusement, il est difficile de progresser dans la résolution de ces problèmes dans le nord car les ressources sont plus limitées. Les gouvernements fédéral et provincial ont bien alloué de nouveaux subsides en 2000 pour s'occuper des sans-abri, mais la part du lion (80 pour cent) est allée aux grandes villes. De petits centres, comme Sudbury, qui comptent beaucoup de sans-abri, sont encore en peine de ressources; pourtant les articles sur les meilleures pratiques à employer pour servir les malades mentaux montrent clairement que des modifications importantes du réseau des services s'imposent pour servir efficacement cette population.

Alors que les Canadiens s'attendent à recevoir les meilleurs traitements et soins de santé et les plus modernes, et s'indignent quand ils n'existent pas, les malades mentaux acceptent sans broncher des services désuets et inférieurs qui sont loin de se classer dans les approches éprouvées. Les malades mentaux sansabri font partie des groupes les plus oppressés de notre société. 
Comme tout le monde, ils ont leurs espoirs et leurs aspirations : un logement stable, un revenu adéquat et des relations sociales harmonieuses. Cependant, les malades mentaux vivent souvent dans des conditions médiocres et ne reçoivent guère plus que des médicaments et un traitement psychiatrique. Il n'est par conséquent pas étonnant, comme l'avance Rapp (1998), qu'ils vivent dans la crainte, l'anxiété, la dépendance, la dépression et l'apathie et, par suite, accèdent difficilement à du soutien. Il est donc vital d'offrir des services et des programmes qui les responsabilisent. Culhans (1992) a relevé certaines caractéristiques clés de services efficaces et adaptés pour les malades mentaux sans-abri : intervention communautaire énergique, logement de transition régi par peu de restrictions et de règlements, offre de services de santé physique et mentale, approche axée sur le consommateur qui tient compte des perspectives et des demandes des clients (y compris l'acceptation de la non-conformité), possibilités d'éducation et de formation professionnelle, aide pour être admissible à un revenu, liens avec des solutions de logement subventionné, souplesse des programmes, et intervention permanente des consommateurs.

Les fournisseurs de services de Sudbury se soucient beaucoup des sans-abri qu'ils servent, se dévouent à leur cause, et s'efforcent de leur offrir les services en tenant compte des restrictions financières sévères, alors que la demande semble dépasser la capacité de servir. Deux séries récentes d'initiatives instaurées dans la collectivité ont attiré l'attention sur les problèmes des sans-abri et de la santé mentale à Sudbury. La première est une enquête sur le décès de Lee Segarra, un jeune homme de 23 ans souffrant d'une grave maladie mentale persistante qui a succombé à l'hypothermie et à de graves engelures en 1998. L'enquête a porté sur les événements entourant cette tragédie, y compris le congé de l'hôpital et les services communautaires, afin de prévenir des accidents semblables à l'avenir (Ontario Ministry of the Solicitor General 2000). La deuxième est l'étude approfondie menée sur les sans-abri de Sudbury. L'attention que le public a porté à ces questions a renforcé les efforts communautaires déployés pour obtenir des ressources supplémentaires pour des programmes, des 
services et des refuges. Des ressources supplémentaires sont fournies pour doubler ou tripler le personnel chargé d'aider les malades mentaux à se loger, pour offrir des services mobiles d'intervention d'urgence, pour dispenser un traitement communautaire dynamique, et pour embaucher d'autres intervenants. À la suite de l'étude documentant la crise locale des sans-abri, les organismes communautaires s'attendent également à recevoir de nouveaux subsides importants ( 2 ou 3 millions de dollars) provenant des gouvernements fédéral et provincial afin de mettre sur pied des initiatives pour les sans-abri.

En outre, les Services sociaux et de santé de la municipalité régionale de Sudbury se sont engagés à financer la reprise de l'étude sur les sans-abri tous les six mois pour les trois prochaines années, notamment un volet sur les dimensions particulières du problème à chaque point de collecte des données. Cette étude nous permettra de suivre l'évolution du problème des sans-abri et de décrire les changements découlant de la hausse des fonds consacrés aux refuges et aux services.

\section{Bibliographie}

ADLAF, E. et Y. ZDANOWICZ (1999), A cluster-analytic study of substance problems and mental health among street youth, American Journal of Drug and Alcohol Abuse, 25 (4), p. 639.

CLARKE INSTITUTE OF PSYCHIATRY (1998), Review of Best Practices in Mental Health Reform, Report prepared for the Federal/Provincial/Territorial Advisory Network on Mental Health 1997, Ottawa, Minister of Public Works and Government services Canada.

COX, G., D.WALKER, S. FRENG, B. SHORT, S. MEIJER et L. GILCHRIST (1998), Outcome of a controlled trial of the effectiveness of intensive case management for chronic public inebriates, Journal of Studies on Alcohol, 59 (5), p. 523.

CULHANS, D. (1992), Ending homelessness among women with severe mental illness: A model program from Philadelphia, Psychosocial Rehabilitation Journal, 16 (1), p. 63.

DALY, G. (1988), A Comparative Assessment of Programs Dealing With the Homeless Population in the United States, Canada, and Britain, Canada Mortgage and Housing Corporation, Ottawa.

DENNIS, M. (1993), The Homeless and Transient Population Study, Research Triangle Park, North Carolina.

DUNPHY, N., L. LAPOINTE et I. DEJONG (1999), Where's Home Part 2? A Picture of Housing Needs in Ontario, Ontario Non-Profit Housing Association \& Co-Operative Housing Federation of Canada Carleton Printers, Toronto.

FISK, D., M. ROWE, D. LAUB, L. CALVOCORESSI et K. DEMINO (2000), Homeless persons 
with mental illness and their families: Emerging issues for clinical work, Families in Society: The Journal of Contemporary Human Services, 81 (4), p. 351.

GRIMM, K. et J. MALDONADO (1995), No home of her own: gender and homelessness, Women E Environments, 14 (2), p. 20.

LEVY,J. (2000), Homeless outreach: On the road to pretreatment alternatives, Families in Society: The Journal of Contemporary Human Services, 81 (4), p. 360.

MAYOR'S HOMELESSNESS ACTIONTASK FORCE (1999), Taking Responsibility for Homelessness: An Action Plan for Toronto, City of Toronto.

MENTAL HEALTH POLICY RESEARCH GROUP (1997), Mental illness and pathways to homelessness: proceedings and recommendations, Canadian Mental Health Association.

MOBRAY, C. et D. BYBEE (1998), The importance of context in understanding homelessness and mental illness: Lessons learned from a research demonstration project, Research on Social Work Practice, 8 (2), p. 172.

OAKELY, D. et D. DENNIS (1996), Responding to the Needs of Homeless People with Alcohol, Drug, and I or Mental Disorders, Washington, D.C., Oryx Press.

ONTARIO MEDICAL ASSOCIATION COMMITTEE ON POPULATION HEALTH (1996), Exploring the health impact on homelessness, Ontario Medical Review, May, www.oma.org/ phealth/homeless.htm

ONTARIO MINISTRY OF THE SOLICITOR GENERAL (2000), Inquest in the Death of Lee Segarra Announced, Press release, Sudbury, August 5, 2000.

PERESSINI, T., L. McDONALD et D. HULCHANSKI (1996), Estimating Homelessness: Towards a Methodology for Counting the Homeless in Canada, Canada Mortgage and Housing Corporation, Ottawa, Ontario.

PUBLIC HEALTH RESEARCH, EDUCATION \& DEVELOPMENT PROGRAM (2000), Report on the Health Status of the Residents of Ontario, http://www.opha.on.ca/specialreports/

RAPP. C. (1998), The Strengths Model: Case Management with People Suffering from Severe and Persistent Mental Illness, Oxford University Press, New York.

UNGER, J., M. KIPKE, T. SIMON, S. MONTGOMERY et C. JOHNSON (1997), Homeless youth and young adults in Los Angeles: Prevalence of mental health problems and the relationship between mental health and substance disorders, American Journal of Community Psychology, 25 (3), p. 371.

VAN DE SANDE, A., J.-M. BÉLANGER, C. KAUPPI, B. MOXAM et R. SANDERSON (1999), Child \& Youth Poverty in Sudbur, Child Poverty Network, Laurentian University, Sudbury.

\section{Note}

1. Ce calcul se fonde sur le dénombrement des 407 personnes et sur l'estimation des sans-abri occultés $(n=273$ ) découlant de l'enquête dans les quartiers. 